\title{
Genetics and public health
}

Peter Harper

$\mathrm{G}$ enetic disorders and their prevention have become one of the principal challenges in medicine. As a clinical geneticist I am excited by the major advances in isolating major human disease genes and am proud that we are able to apply these rapidly and often effectively to help families with serious genetic disorders. I feel uneasy, however, when I look at the future possibilities for misuse of these advances. In the past few years community genetics has emerged. This represents the population based rather than individual family oriented aspects of medical genetics, notably screening programmes for genetic disorders and for congenital malformations in pregnancy. The proliferation of such programmes demands coordination and initial evaluation and I have been among those who have supported this. So why am I ambivalent and worried?

Preclinical testing for genetic disorders is not new, but the development of an increasing number of accurate and totally age independent tests for genetic disorders is going to pose major problems, which have already been encountered for disorders such as Huntington's disease. At present such tests are mainly undertaken in the context of family risk, but the increasing possibility of testing for specific mutations is shifting their use towards a diagnostic and population basis. Should we test all apparently sporadic cases of a disorder such as Alzheimer's disease to detect the minority that are caused by inherited mutations? The response of the public health physician might depend on the yield of such a test (currently unknown), but of greater importance might be the effect on the family of identifying a serious risk to the mental health of relatives in later life when this was previously unsuspected. If screening for such mutations becomes a part of regular practice it will have major implications, comparable with those of screening for HIV.

The only molecular test to have actually entered the population screening arena is that for the common cystic fibrosis mutation, which is carried by around one in 35 individuals in populations of northern European origin. Here some of the problems are lessened by the recessive nature of the disease-carriers are and will remain clinically normal-but will the perception of those detected reflect this?

Experience from haemoglobinopathy and Tay-Sachs carrier screening suggests that avoidance of stigmatisation and other problems depends on the effectiveness of education regarding the disorder and its carrier status. Is our population sufficiently educated to be able to handle this information fruitfully? It will be important for this to be resolved by the current pilot projects before any general programme is accepted as a service.

Occupational testing is an area where molecular testing may in future impinge directly on public health medicine. As susceptibility genes are progressively identified for common cancers, cardiovascular disorders, and mental illness should they be tested for in the context of environmental hazards of stress or particular occupations? If so, how will such information be used by employers and others? An allied and more immediate problem relates to the use of genetic testing in life and health insurance.

\section{Future developments should be discussed and debated openly, involving the public as well as professionals.}

Again the experience of HIV testing in this context provides an important and none too encouraging lesson.

Testing children for late onset genetic disorders is a subject that has worried my colleagues and me. I can think of no more important issue which has been so ignored by those professionals who ought to be most concerned. At present this problem arises principally in the context of individual family risk, but the potential for population applications already exists. Every baby has a filter paper blood spot taken for detecting such treatable disorders as phenylketonuria and hypothyroidism, but what rules should govern its use for detecting late onset and untreatable disorders? The potential for harm resulting from the ill considered testing of children for adult genetic disorders is great, yet most clinical colleagues are reluctant to admit it as a major issue.

How can we best prevent future problems?

- We need to distinguish between population and individual aims. Population prevention of a serious genetic disorder-for example, Huntington's disease -is a valid goal to aim for, but it is different from the aim of genetic counselling and any associated predictive testing, where the goal is to help a specific individual, couple, or family achieve what is the optimal decision for themselves. To avoid such possible conflicts of interest it could be argued that those undertaking the individual genetic counselling of families should not be those also running population based programmes. I would recommend the reverse, since each will gain insight by being exposed to the potentially opposing point of view. Thus if any defined field or subspecialty of community genetics does develop I think that those concerned will benefit by having some commitment to seeing and counselling individual families rather than simply regarding them as numbers.

- To avoid problems in the future we need to be aware of the past. Most of those working in medical genetics (including me until recently) are ignorant of the past abuse of our subject. It is easy to regard the excesses of the eugenics movement, or the abuses in Nazi Germany, as disconnected from present day medical genetics, but a closer look at these chapters shows that their key feature was the subordination of individual decisions to the broader population based goals. That these goals were often deeply flawed is not the relevant point. Those concerned at the time believed that they were acting in the best interests of the present and future population and that these must override the lesser rights and decisions of individuals. There is a clear danger here for how we judge the success of any population based genetic programmes, and for conflict with any individual decisions that seem to jeopardise such success.

- Future developments should be discussed and debated openly, involving the public as well as professionals. A major problem in carrying this out in Britain, as in most countries, is the poor state of education of the public regarding science in general and genetics in particular. The tendency of the media to highlight the sensational and threatening aspects does not help. It is therefore important for those engaged in community genetics to undertake such education in relation to screening programmes and the broad area of genetic disorders before new programmes are introduced.

The field of medical genetics is now at a stage where it has the potential for influencing whole populations, not just families at high risk. The potential for harm is correspondingly great if we do not think carefully about the effects of new developments. We should be able to avoid most of the serious problems if we do not forget the individuals who make up all populations. Any applications of genetics in public health need to retain the respect for the autonomy of individuals and for the inevitable variation in decisions that people make. It will be a challenge during the coming years for medical geneticists and those in public health to combine their respective skills so that individual families and entire populations benefit from the discoveries in genetics that are likely to continue and even increase in pace over the next decade.-PETER HARPER is a physician and professor of medical genetics in Cardiff 